\title{
UDC 547.512;535.338
}

\section{SYNTHESIS OF ETHYL ESTERS OF PHENYL- AND $p$-PHENYL SUBSTITUTED CYCLOPROPANECARBOXYLIC ACIDS AND THEIR CONVERSION}

\author{
K.G.Guliyev, A.E.Rzayeva, A.M.Guliyev \\ Institute of Polymer Materials NAS of Azerbaijan \\ guliyev.kazim_pm@mail.ru \\ Received 09.06.2020 \\ Accepted 23.09.2020
}

\begin{abstract}
The reaction of carboethoxycarbene with styrene and $p$-substituted styrenes in the conditions of thermocatalytic decomposition of ethyl diazoacetate has been studied. It has been shown that the substituents in the investigated styrenes have an essential effect on the activity of the double bond with respect to carboethoxycarbene. It has been established as a result of the investigation that the substituents in the para-position of styrene influence essentially on the activity of the double bond with respect to carboethoxycarbene. It has been revealed that carboethoxycarbene is connected to the double bond of styrene and $p$-styrenes practically without formation of by products. As a result of the reaction, 2-phenyl and 2-p-substituted phenyl-1ethoxycarbonyl cyclopropane (1-4) as a mixture of cis- and trans- isomers at a ratio of (30:70) with the predominant formation of a trans-isomer has been obtained. The composition and structure of the synthesized compounds have been established. It has been revealed that all synthesized cyclopropane-containing compounds show the various biologically active properties. In addition, the compounds 9-12 can be used as a modifier and diluent for epoxy resin ED-20.
\end{abstract}

Keywords: cyclopropane, p-substituted styrene, carboethoxycarbene, glycidyloxymethylcyclopropane.

doi:

\section{Introduction}

Functionally substituted cyclopropanes are one of the leading sections of the organic chemistry, constantly attracting higher interest of researchers. This has been stipulated by both large synthetic and theoretical value of the cyclopropane derivatives and by the fact that they exhibit various biological activity [1-4]. It has been revealed in carrying out of the systematic investigations that the polymers obtained on the basis of cyclopropane derivatives have high photosensitivity [5-9]. The systematic investigations of the reaction of functionally substituted cyclopropanes, study of the composition, structure and properties of these cyclopropanecontaining compounds have been presented.

In this work the activity of the double bonds of styrene and para-substituted styrene ( $p$-methyl, $p$-chloro- and $p$-nitro styrenes) with respect to carboethoxycarbene has been studied. The reactions of carboethoxycarbene with styrene and $p$-substituted styrenes were carried out with the use of a twofold excess of styrenes and substituted styrenes under the conditions of thermo-catalytic ( $\mathrm{CuSO}_{4}$ anhydrous) decomposition of ethyl diazoacetate.
It has been established as a result of the investigation that the substituents in the paraposition of styrene influence essentially on the activity of the double bond with respect to carboethoxycarbene. It has been revealed that carboethoxycarbene is connected to the double bond of styrene and $p$-styrenes practically without formation of by products. As a result of the reaction 2-phenyl- and 2-p-substituted phenyl1-ethoxycarbonyl cyclopropane (1-4) as a mixture of cis- and trans-isomers at a ratio of $\sim(30: 70)$ with the predominant formation of a trans-isomer has been obtained.

In all cases, as expected, the electrondonor substituents increase the reactivity of the double bond, and the electron-acceptor ones decrease it. Indeed, the ethyl ester of fumaric and maleic acids and other side substances in the reaction mixture have been not practically found.

The yields of esters (1-4) largely depend on the reaction conditions and the substituent in the para-position of the styrenes and are on average $65-96 \%$. It was shown that an introduction of a methyl group into phenyl nucleus, i.e. a transition from styrene to $p$-methylstyrene leads to the expected increase in reactivity. 
At restoration of a mixture of isomers of esters 1-4 the corresponding hydroxymethyl substituted phenylcyclopropanes have been obtained. The synthesis of compounds 5-8 was carried out under action of $\mathrm{LiAlH}_{4}$ on $1-4$ in boiling diethyl ether converted $82-95 \%$ yields to the corresponding carbinols 5-8. The high selectivity of $\mathrm{LiAlH}_{4}$, used as a restoring reagent, allows to carry out the restoration reactions only on the carbonyl group of the initial ester 1-4, leaving the cyclopropane group unaffected. Consequently, a ratio of stereoisomers at restoration remained practically unchanged, that allows to confirm - the change of configuration of the initial mixture of isomers does not take place.

The synthesis of compounds 9-12 was carried out by interaction reaction of the compounds 5-8 with epichlorohydrin in the present $\mathrm{NaOH}$ according to the reaction:

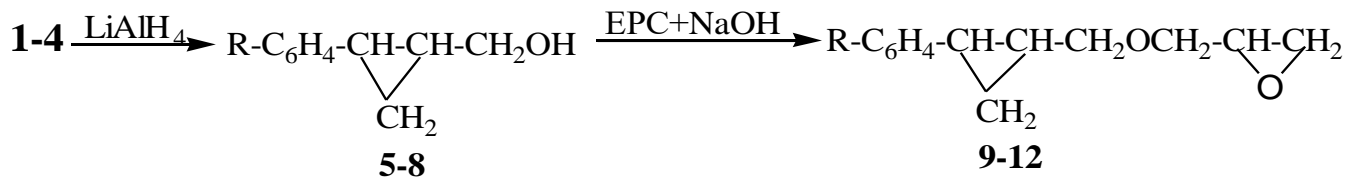

$\mathrm{R}=\mathrm{H}(5), \mathrm{CH}_{3}\left({ }_{6}\right), \mathrm{Cl}\left({ }_{7}\right), \mathrm{NO}_{2}\left({ }_{8}\right)$
$\mathrm{R}=\mathrm{H}(9), \mathrm{CH}_{3}\left({ }_{10}\right), \mathrm{Cl}\left({ }_{11}\right), \mathrm{NO}_{2}\left({ }_{12}\right)$

A degree of purity of the compounds 1-12 $(99.8-99.9 \%)$ has been determined by GLC on chromatograph LCM-8 MD. All synthesized compounds 1-12 have been characterized by data of elemental analysis, IR spectra NMR and Figure $(1,2)$. In the IR spectra of all synthesized compounds there are the absorption bands in the field of $1570-1620 \mathrm{~cm}^{-1}, 1450-1500 \mathrm{~cm}^{-1}$ and $3000-3030 \mathrm{~cm}^{-1}$ characteristic for valence vibrations of the benzene ring, the bands of low intensity in the field of $1000-1100 \mathrm{~cm}^{-1}$, presenting planar deformation vibrations of $\mathrm{C}-\mathrm{H}$ of the same ring and intensive bands in the field of 690-750 $\mathrm{cm}^{-1}$; characteristic for non-planar deformation vibrations of a three-membered cycle in the field of $3040-3045 \mathrm{~cm}^{-1}(\mathrm{C}-\mathrm{H}$ valence vibration) and in the field of $1010-1030 \mathrm{~cm}^{-1}$ (vibration of $\Delta$-cycle itself). In addition, in the IR spectra of the compounds $3,7,11$, there are the absorption bands at $750-760 \mathrm{~cm}^{-1}$ characteristic for the chlorine atom and bands at 1335 and $1525 \mathrm{~cm}^{-1}$, related to vibrations for $\mathrm{NO}_{2}$ of the compounds $4,8,12$. It has been revealed that the absorption bands of the benzene and cyclopropane rings are retained in the compounds 9-12 and there are the absorption bands at 875 and $1250 \mathrm{~cm}^{-1}$ for $\mathrm{C}^{-\mathrm{C}-\mathrm{C}-}$ fragment.

In the IR spectra of 2-phenylcyclopropylcarbinols (5-8) in the pure form, it is seen a strong absorption in the field of $\sim 1050 \mathrm{~cm}^{-1}$, which is probably characteristic for alcohols $\mathrm{C}-$ O group. The absorption of alcohols 5-8 is especially appeared as two broad bands: one at $3340 \mathrm{~cm}^{-1}$ and the second, relatively lowintensive at $3560 \mathrm{~cm}^{-1}$. The first is usually referred to the valence vibration of hydroxyl groups included in "polymer complexes". The second - the vibration of $\mathrm{OH}$ groups forming the dimer ring. At the solution of carbinols 5-8 are dissolved in $\mathrm{CCl}_{4}$, the band at $3340 \mathrm{~cm}^{-1}$ shifts to $\sim 3360 \mathrm{~cm}^{-1}$, and it is simultaneously appeared a low-intensive band at $3640 \mathrm{~cm}^{-1}$. This fact indicates that at dissolution in $\mathrm{CCl}_{4}$ $(\sim 30 \%)$ a small number of alcohol molecules is released from hydrogen bond and is in a state of monomers. Indeed, at further dilution of the solution, an intensity of the band at $3640 \mathrm{~cm}^{-1}$ is increased, that corresponds to an increase of a number of monomeric alcohol molecules in the solution. During the study of the spectra of alcohols $5-8$ in a dioxane solution $(40 \%)$, it has been revealed that the absorption band at $3340 \mathrm{~cm}^{-1}$ is shifted toward the waves up to $3500 \mathrm{~cm}^{-1}$.

It has been detected that an increase of dioxane in the solution leads to a decrease of the band intensity at $3500 \mathrm{~cm}^{-1}$ and it is simultaneously appeared a low-intensity band at $3620 \mathrm{~cm}^{-1}$. 


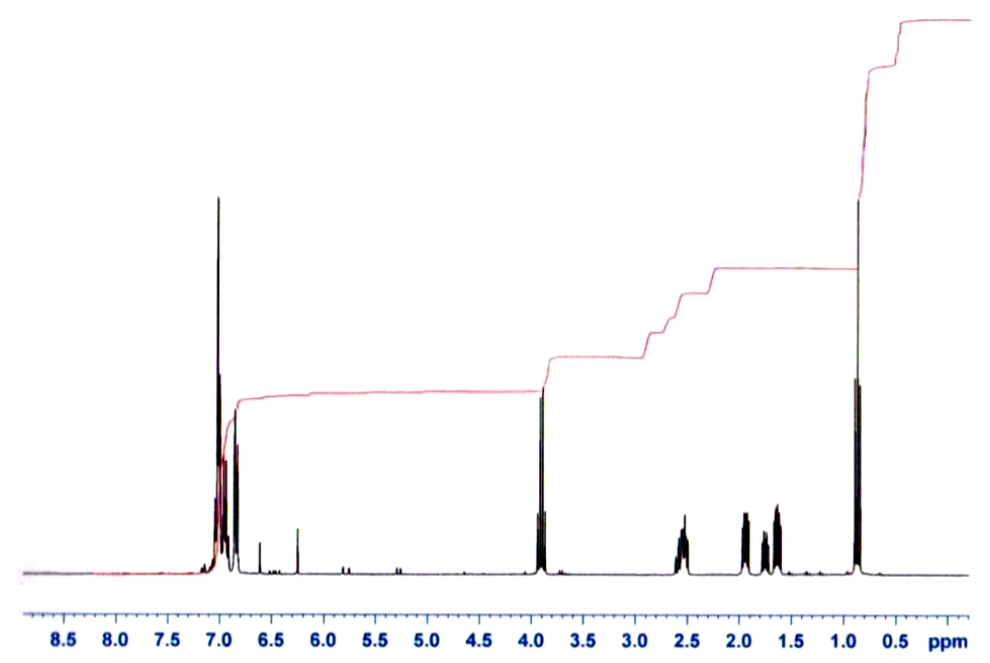

Fig. 1. NMR spectra 2-phenyl-1-hydroxymethylcyclopropane.

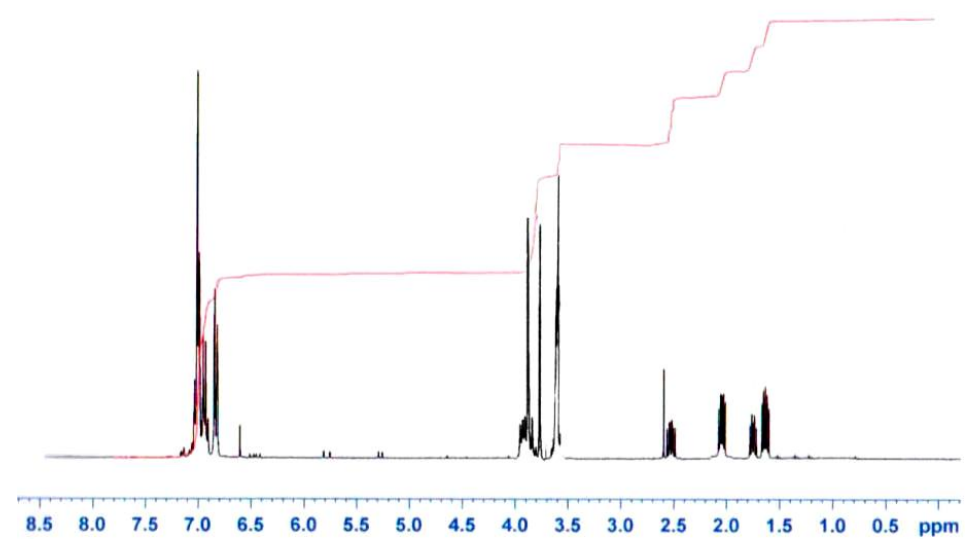

Fig. 2. NMR spectra 2-phenyl-1-ethoxycarbonylcyclopropane.

The band at $3500 \mathrm{~cm}^{-1}$ which can be attributed to the vibration $-\mathrm{OH}$ group connected to the dimeric ring of type ${ }^{\mathrm{R}-\mathrm{O}<}<_{\mathrm{H}^{-}}^{\mathrm{H}_{-}>-\mathrm{O}-\mathrm{R}}$. Probably, when the investigated alcohol is dissolved in such a strongly polar solvent as dioxane, a possibility of the formation of a hydrogen bond between dioxane and alcohol molecules, the vibration of hydroxyl group, which in this case appears around $3500 \mathrm{~cm}^{-1}$, cannot be ruled out.

The observed band at $3500 \mathrm{~cm}^{-1}$ in the spectra of a dioxane solution of 2-phenylcyclopropylcarbinols (5-8), is probably referred to the vibrations of hydroxyl groups forming hydrogen bonds between alcohol molecules in dimeric complexes and between alcohol molecules in dioxane. The bands at $3620 \mathrm{~cm}^{-1}$, which appear as a result of some decrease of the concentration of closed dimeric complexes at dilution, probably can be attributed to the vibration of end hydroxyl group of the dimer type R-H--.-- $\mathrm{R}-\mathrm{H}$.

It has been revealed that all synthesized cyclopropane-containing compounds exhibit various biologically active properties. In addition, the compounds 9-12 can be used as a modifier and diluent for epoxy resin ED-20.

\section{Experimental part}

The IR spectra have been recorded on an "Agilent Cary 630 FTIR" apparatus in a thin layer. The ${ }^{1} \mathrm{H}$ NMR spectra have been obtained on a Bruker AFR-300 spectrometer in $\mathrm{CDCl}_{3}$, the chemical shifts have been determined rela- 
tively tetramethylsilane (TMS). The purity of the synthesized compounds was controlled by methods of GLC and TLC on UV-254 plates, and detected by UV light or iodine vapors.

1. Reaction of carboethoxycarbene with phenyl-substituted styrenes. General method. A mixture of $0.6 \mathrm{~mol}(62.4 \mathrm{~g})$ of styrene or pphenyl substituted styrene and $0.2 \mathrm{~g}$ of anhydrous copper sulfate as a catalyst was heated to $40-50^{\circ} \mathrm{C}$ and in stirring $0.5 \mathrm{~mol}$ of ethyl diazoacetate was added dropwise (addition rate 10$12 \mathrm{~g} / \mathrm{h}$ ). After addition of all of the ethyl diazoacetate and stopping the isolation of nitrogen, the reaction mixture was cooled to room temperature. The catalyst $\mathrm{CuSO}_{4}$ was filtered off, the filtrate was extracted several times with diethyl ether and the resulting reaction mass was combined. Further diethyl ether was distilled and then was distilled in vacuum. At the beginning - an excess of styrene or phenyl substituted styrene, and then - main fraction 1-4.

The IR spectra of the compounds 1-4 contain the absorption bands in the field of 3095-3100, 1030-1035 ( $\left.v_{\mathrm{C}-\mathrm{H} \text { cycle }}\right), 2980,2940$, 2880 ( $v_{\mathrm{C}-\mathrm{H}}$ aliph. $), 750-760\left(v_{\mathrm{Cl}}\right), 1690,1720$ $\left(v_{\mathrm{C}=\mathrm{O}}\right), 1450-1500 \mathrm{~cm}^{-1}$ and $1570-1620 \mathrm{~cm}^{-1}$, 1335 and $1525 \mathrm{~cm}^{-1}\left(\mathrm{NO}_{2}\right)$ of the compound 4 . The bands $690-750 \mathrm{~cm}^{-1}$ are referred, to out-ofplanar deformation vibrations of $\mathrm{C}-\mathrm{H}$ in monosubstituted benzene of the compound 1 .

2-phenyl-1-ethoxycarbonylcyclopropane (1). Formula $\mathrm{C}_{12} \mathrm{H}_{14} \mathrm{O}_{2}$. Yield - 90\%, b.p. $68-70^{\circ} \mathrm{C}(3 \mathrm{~mm} \mathrm{Hg}), n_{D}^{20}=1.5125, d_{4}^{20}=1.08$, $M R_{D}=50.713 .{ }^{1} \mathrm{H}$ NMR spectrum, $\delta$, ppm: $6.95 \mathrm{~m}\left(5 \mathrm{H}_{\text {arom }}\right), 1.92 \mathrm{~m}\left(1 \mathrm{H}, \mathrm{CH}_{\text {cycloprop. }}\right), 2.56$ m (1H, $\left.\mathrm{CH}_{\text {cycloprop. }}\right), 1.65 \mathrm{~m}\left(1 \mathrm{H}, \mathrm{CH}_{2 \text { cycloprop. }}\right)$, $1.62 \mathrm{~m}\left(1 \mathrm{H}, \mathrm{CH}_{2 \text { cycloprop. }}\right), 3.85 \mathrm{q}\left(2 \mathrm{H}, \mathrm{CH}_{2}\right)$, $0.96 \mathrm{t}\left(3 \mathrm{H}, \mathrm{CH}_{3}\right)$. Found, \%: C 75.20, H 7.91. Calculated, \%: C 75.79, H 7.37.

2-p(methylphenyl)-1-ethoxycarbonylcyclopropane (2). Formula $\mathrm{C}_{13} \mathrm{H}_{16} \mathrm{O}_{2}$. Yield - 95\%, b.p. $\quad-75-77^{\circ} \mathrm{C} \quad(3 \mathrm{~mm} \mathrm{Hg}), \quad n_{D}^{20}=1.500$, $d_{4}^{20}=1.06, M R_{D}=55.331 .{ }^{1} \mathrm{H}$ NMR spectrum, $\delta$, ppm: $7.00 \mathrm{~m}\left(4 \mathrm{H}_{\text {arom. }}\right), 1.00 \mathrm{~s}\left(\mathrm{CH}_{3}\right), 1.93 \mathrm{~m}$ (1H, $\left.\mathrm{CH}_{\text {cycloprop. }}\right), 2.58 \mathrm{~m}\left(1 \mathrm{H}, \mathrm{CH}_{\text {cycloprop. }}\right), 1.64$ $\mathrm{m}\left(1 \mathrm{H}, \mathrm{CH}_{2 \text { cycloprop }}\right), 1.65 \mathrm{~m}\left(1 \mathrm{H}, \mathrm{CH}_{2 \text { cycloprop }}\right)$, $3.92 \mathrm{q}\left(2 \mathrm{H}, \mathrm{CH}_{2}\right), 1.05 \mathrm{t}\left(3 \mathrm{H}, \mathrm{CH}_{3}\right)$. Found, \%: C 76.58, H 7.20. Calculated, \%: C 76.47, H 7.84. 2-p(chlorophenyl)- 1-ethoxycarbonylcyclopropane (3). Formula $\mathrm{C}_{12} \mathrm{H}_{13} \mathrm{O}_{2} \mathrm{Cl}$. Yield $82 \%$, b.p. $-85-87^{0} \mathrm{C}(3 \mathrm{~mm} \mathrm{Hg}), n_{D}^{20}=1.5640$, $d_{4}^{20}=1.32, M R_{D}=55.58 .{ }^{1} \mathrm{H} \mathrm{NMR}$ spectrum, $\delta$, ppm: $7.10 \mathrm{~m}\left(4 \mathrm{H}_{\text {arom }}\right), 2.08 \mathrm{~m}\left(1 \mathrm{H}, \mathrm{CH}_{\text {cyclo- }}\right.$ prop.), $2.55 \mathrm{~m}\left(1 \mathrm{H}, \mathrm{CH}_{\text {cycloprop. }}\right), 1.71 \mathrm{~m}(1 \mathrm{H}$, $\left.\mathrm{CH}_{2 \text { cycloprop. }}\right), 1.65 \mathrm{~m}\left(1 \mathrm{H}, \mathrm{CH}_{2 \text { cycloprop. }}\right), 4.00 \mathrm{q}$ $\left(2 \mathrm{H}, \mathrm{CH}_{2}\right), 1.0 \mathrm{t}\left(3 \mathrm{H}, \mathrm{CH}_{3}\right)$. Found, \%: $\mathrm{C} 64.28$, $\mathrm{H}$ 5.32, $\mathrm{Cl}$ 15.75. Calculated, \%: $\mathrm{C}$ 64.14, $\mathrm{H}$ 5.79, Cl 15.81 .

2-p(nitrophenyl)- 1-ethoxycarbonylcyclopropane (4). Formula $\mathrm{C}_{12} \mathrm{H}_{13} \mathrm{O}_{4} \mathrm{~N}$. Yield $65 \%$, b.p. $\quad-140-142^{\circ} \mathrm{C} \quad(3 \mathrm{~mm} \mathrm{Hg})$, $n_{D}^{20}=1.5610, d_{4}^{20}=1.30 .{ }^{1} \mathrm{H}$ NMR spectrum, $\delta$, ppm: $7.50 \mathrm{~m}\left(4 \mathrm{H}_{\text {arom. }}\right), 2.09 \mathrm{~m}\left(1 \mathrm{H}, \mathrm{CH}_{\text {cyclo- }}\right.$ prop.), $2.68 \mathrm{~m}\left(1 \mathrm{H}, \mathrm{CH}_{\text {cycloprop. }}\right), 1.67 \mathrm{~m}(1 \mathrm{H}$, $\left.\mathrm{CH}_{2 \text { cycloprop. }}\right), 1.63 \mathrm{~m}$ (1 H, $\left.\mathrm{CH}_{2 \text { cycloprop. }}\right), 4.13 \mathrm{q}$ $\left(2 \mathrm{H}, \mathrm{CH}_{2}\right), 1.12 \mathrm{t}\left(3 \mathrm{H}, \mathrm{CH}_{3}\right)$. Found, \%: C 61.11, H 5.63, N 5.80. Calculated, \%: C 61.28, H 5.53, N 5.95.

2. Synthesis of 2-p-phenyl-substituted-1hydroxymethylyclopropanes. General method. $9.5 \mathrm{~g}(0.25 \mathrm{~g} \cdot \mathrm{mol}) \mathrm{LiAlH}_{4}$ in $200 \mathrm{ml}$ of absolute diethyl ether was placed to a three-necked flask with a capacity of $500 \mathrm{ml}$, equipped with a mechanical stirrer, a dropping funnel and a reflux condenser and was added $0.25 \mathrm{~mol}(47.5 \mathrm{~g} \cdot \mathrm{mol})$ of ethyl ester of 2-phenylcyclopropane-1-carboxylic acid for 2-2.5 hours. With this the temperature of the reaction medium was maintained at $25-35^{\circ} \mathrm{C}$. After addition the whole initial ester, the stirring was continued for another 30 minutes. Then, the distilled water was added dropwise into the flask, and then $-5 \%$ solution of hydrochloric acid. The ether layer was separated; the aqueous layer was extracted with ether (twice in $20 \mathrm{ml}$ ). The ether extracts were combined with the ether layer and dried over calcined $\mathrm{Na}_{2} \mathrm{SO}_{4}$. After distilling off the sulfuric ether, the reaction products were distilled under vacuum. 6-8 was similarly synthesized.

The IR spectra of the compounds 5-8 contain the absorption bands in the field of

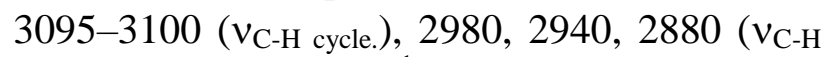
aliph.), 2900-3100 cm $\mathrm{cm}^{-1}(\mathrm{OH}), 758-770\left(v_{\mathrm{Cl}}\right)$, 1335 and $1525 \mathrm{~cm}^{-1}\left(\mathrm{NO}_{2}\right)$. 
2-phenyl-1-hydroxymethylcyclopropane (5). Formula $\mathrm{C}_{10} \mathrm{H}_{12} \mathrm{O}$. Yield - 92\%, b.p. $-125-$ $130^{\circ} \mathrm{C}(9 \mathrm{~mm} \mathrm{Hg}), n_{D}^{20}=1.5110, d_{4}^{20}=1.335$, $M R_{D}=43.235 .{ }^{1} \mathrm{H}$ NMR spectrum, $\delta$, ppm: $6.92 \mathrm{~m}\left(5 \mathrm{H}_{\text {arom. }}\right), 1.93 \mathrm{~m}\left(1 \mathrm{H}, \mathrm{CH}_{\text {cycloprop. }}\right), 2.61$ m $\left(1 \mathrm{H}, \mathrm{CH}_{\text {cycloprop. }}\right), 1.63 \mathrm{~m}\left(1 \mathrm{H}, \mathrm{CH}_{2 \text { cycloprop. }}\right)$, $1.65 \mathrm{~m}\left(1 \mathrm{H}, \mathrm{CH}_{2 \text { cycloprop. }}\right), 3.86 \mathrm{~s}\left(2 \mathrm{H}, \mathrm{CH}_{2} \mathrm{O}\right)$, $3.64 \mathrm{~s}(1 \mathrm{H}, \mathrm{OH})$. Found, \%: C 81.25, H 8.05. Calculated, \%: C 81.08, H 8.10.

2-p(methylphenyl)1-hydroxymethylcyclopropane (6). Formula $\mathrm{C}_{11} \mathrm{H}_{14} \mathrm{O}$. Yield - 93\%, b.p. $-130-137^{0} \mathrm{C}(9 \mathrm{~mm} \mathrm{Hg}), n_{D}^{20}=1.5130$, $d_{4}^{20}=1.350, M R_{D}=46.920 .{ }^{1} \mathrm{H}$ NMR spectrum, $\delta$, ppm: $6.95 \mathrm{~m}\left(4 \mathrm{H}_{\text {arom. }}\right), 0.95 \mathrm{~s}(3 \mathrm{H}$, $\left.\mathrm{CH}_{3}\right), 1.94 \mathrm{~m}\left(1 \mathrm{H}, \mathrm{CH}_{\text {cycloprop. }}\right), 2.63 \mathrm{~m}(1 \mathrm{H}$, $\left.\mathrm{CH}_{\text {cycloprop. }}\right), 1.66 \mathrm{~m}\left(1 \mathrm{H}, \mathrm{CH}_{2 \text { cycloprop. }}\right) 1.68 \mathrm{~m}$ $\left(1 \mathrm{H}, \mathrm{CH}_{2 \text { cycloprop. }}\right), 3.87 \mathrm{~d}(2 \mathrm{H}, \mathrm{CH} 2 \mathrm{O}), 3.66 \mathrm{~s}$ $(1 \mathrm{H}, \mathrm{OH})$. Found, \%: C 81.59, H 8.83. Calculated, \%: C 81.48, H 8.64.

2-p(chlorophenyl)1-hydroxymethylcyclopropane (7). Formula $\mathrm{C}_{10} \mathrm{H}_{11} \mathrm{OCl}$. Yield $94 \%$, b.p. $\quad-135-142^{\circ} \mathrm{C} \quad(9 \mathrm{~mm} \quad \mathrm{Hg})$, $n_{D}^{20}=1.5225, d_{4}^{20}=1.478, M R_{D}=48.998 .{ }^{1} \mathrm{H}$ NMR spectrum, $\delta$, ppm: $7.14 \mathrm{~m}\left(4 \mathrm{H}_{\text {arom. }}\right), 2.05$ $\mathrm{m}\left(1 \mathrm{H}, \mathrm{CH}_{\text {cycloprop. }}\right), 2.57 \mathrm{~m}\left(1 \mathrm{H}, \mathrm{CH}_{\text {cycloprop. }}\right)$, $1.66 \mathrm{~m} \quad\left(1 \mathrm{H}, \mathrm{CH}_{2 \text { cycloprop. }}\right), 1.72 \mathrm{~m}(1 \mathrm{H}$,

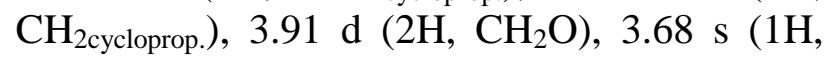
$\mathrm{OH})$. Found, \%: C 65.20, H 6.75, Cl 19.94. Calculated, \%: C 65.75, H 6.03, Cl 19.45 .

2-p(nitrophenyl)1-hydroxymethylcyclopropane (8). Formula $\mathrm{C}_{10} \mathrm{H}_{11} \mathrm{O}_{3} \mathrm{~N}$. Yield $93 \%$, b.p. $\quad 131-149^{\circ} \mathrm{C} \quad(2 \mathrm{~mm} \mathrm{Hg})$, $n_{D}^{20}=1.5190, d_{4}^{20}=1.524, M R_{D}=49.998 .{ }^{1} \mathrm{H}$ NMR spectrum, $\delta$, ppm: $7.55 \mathrm{~m}\left(4 \mathrm{H}_{\text {arom. }}\right), 2.17$ m (1 H, $\left.\mathrm{CH}_{\text {cycloprop. }}\right), 2.60 \mathrm{~m}$ (1 H, $\left.\mathrm{CH}_{\text {cycloprop. }}\right)$, $1.65 \mathrm{~m}$ (1 H, CH 2cycloprop. $), 1.69 \mathrm{~m}$ (1 H, $\left.\mathrm{CH}_{2 \text { cycloprop. }}\right), 3.98 \mathrm{~d}\left(2 \mathrm{H}, \mathrm{CH}_{2} \mathrm{O}\right), 3.72 \mathrm{~s}(1 \mathrm{H}$, $\mathrm{OH}$ ). Found, \%: C 62.22, H 5.40, N 7.80. Calculated, \%: C 62.18, H 5.69, N 7.25.

3. Synthesis of p-substituted 2-phenyl1-glycidyloxymethylcyclopropane. General method. $14.8 \mathrm{~g}(0.1 \mathrm{~mol})$ of 2-phenyl-1-hydroxymethylcyclopropane in $50 \mathrm{ml}$ of absolute diethyl ether, $0.2 \mathrm{~g}$ of hydroquinone and $8 \mathrm{~g}$ $(0.2 \mathrm{~mol})$ of powdered caustic sodium were placed to a three-necked flask; then with intensive mixing $15.3 \mathrm{~g}(0.2 \mathrm{~mol})$ of epichlorohydrin was added. The reaction mixture was stirred for $4 \mathrm{~h}$, the precipitated $\mathrm{NaCl}$ was filtered. After this, the diethyl ether was firstly distilled off. The residue was distilled in a vacuum. 10-12 was similarly synthesized.

The IR spectra of the compounds 9-12 contain the absorption bands in the field of 1435-1440 $\mathrm{cm}^{-1}, 3095-3100 \mathrm{~cm}^{-1}$ ( $\left.v_{\mathrm{C}-\mathrm{H} \text { cycle }}\right)$, $2880 \mathrm{~cm}^{-1}, 2940 \mathrm{~cm}^{-1}, 2980 \mathrm{~cm}^{-1}\left(v_{\mathrm{C}-\mathrm{H}}\right.$ aliph. $)$, $755-770\left(v_{\mathrm{Cl}}\right), 1365$ and $1335 \mathrm{~cm}^{-1}\left(\mathrm{NO}_{2}\right), 875-$ $1250 \mathrm{~cm}^{-1}$ ( O $^{-C^{-}}$).

2-phenyl-1-glycidyloxymethylcyclopropane (9). Formula $\mathrm{C}_{13} \mathrm{H}_{16} \mathrm{O}_{2}$. Yield $-95 \%$, b.p. $-140-143^{0} \mathrm{C}(1 \mathrm{~mm} \mathrm{Hg}), n_{D}^{20}=1.520$, $d_{4}^{20}=1.26, M R_{D}=54.895 .{ }^{1} \mathrm{H}$ NMR spectrum, $\delta$, ppm: $7.05 \mathrm{~m}\left(5 \mathrm{H}_{\text {arom }}\right), 1.90 \mathrm{~m}\left(1 \mathrm{H}, \mathrm{CH}_{\text {cyclo- }}\right.$ prop.), $2.57 \mathrm{~m}\left(1 \mathrm{H}, \mathrm{CH}_{\text {cycloprop. }}\right), 1.57 \mathrm{~m}(1 \mathrm{H}$, $\left.\mathrm{CH}_{2 \text { cycloprop. }}\right), 1.60 \mathrm{~m}$ (1 H, $\left.\mathrm{CH}_{2 \text { cycloprop. }}\right), 3.50 \mathrm{~d}$ $\left(2 \mathrm{H}, \mathrm{CH}_{2 \mathrm{eth}}\right), 3.85 \mathrm{~d}\left(2 \mathrm{H}, \mathrm{CH}_{2 \mathrm{ether}}\right), 2.96 \mathrm{~m}(1 \mathrm{H}$, $\left.\mathrm{CH}_{\text {ep. }}\right), 2.30 \mathrm{~d}\left(2 \mathrm{H}, \mathrm{CH}_{2 \text { ep. }}\right)$. Found, \%: C 76.38, H 7.90. Calculated, \%: C 76.47, H 7.84.

2-p(methylphenyl)1-glycidyloxymethylcyclopropane (10). Formula $\mathrm{C}_{14} \mathrm{H}_{18} \mathrm{O}_{2}$. Yield $93 \%$, b.p. $-144-150^{\circ} \mathrm{C} \quad(1 \mathrm{~mm}$ mer.c $)$, $n_{D}^{20}=1.5180, d_{4}^{20}=1.114, M R_{D}=59.098 .{ }^{1} \mathrm{H}$ NMR spectrum, $\delta$, ppm: $7.10 \mathrm{~m}\left(4 \mathrm{H}_{\text {arom. }}\right), 1.10 \mathrm{~s}$ $\left(3 \mathrm{H}, \mathrm{CH}_{3}\right), 1.92 \mathrm{~m}\left(1 \mathrm{H}, \mathrm{CH}_{\text {cycloprop. }}\right), 2.55 \mathrm{~m}$ (1H, $\left.\mathrm{CH}_{\text {cycloprop. }}\right), 1.59 \mathrm{~m}$ (1H, $\left.\mathrm{CH}_{2 \text { cycloprop. }}\right), 1.62$

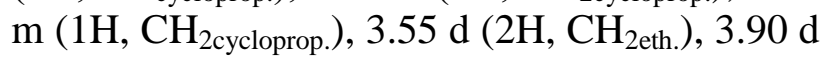
$\left(2 \mathrm{H}, \mathrm{CH}_{2 \text { eth. }}\right), 2.98 \mathrm{~m}\left(1 \mathrm{H}, \mathrm{CH}_{\mathrm{ep}}\right), 2.35 \mathrm{~d}(2 \mathrm{H}$, $\mathrm{CH}_{2 \text { ep. }}$. Found, \%: C 77.45, H 8.13. Calculated, $\%$ : C 77.06, H 8.25.

2-p(chlorophenyl)1-glycidyloxymethylcyclopropane (11). Formula $\mathrm{C}_{13} \mathrm{H}_{15} \mathrm{O}_{2} \mathrm{Cl}$. Yield $-96 \%$, b.p. $-144-147^{\circ} \mathrm{C}(1 \mathrm{~mm} \mathrm{Hg})$ $n_{D}^{20}=1.5230, d_{4}^{20}=1.196, M R_{D}=60.821 .{ }^{1} \mathrm{H}$ NMR spectrum, $\delta$, ppm: $7.16 \mathrm{~m}\left(4 \mathrm{H}_{\mathrm{ph}}\right), 1.87 \mathrm{~m}$ (1H, $\left.\mathrm{CH}_{\text {cycloprop. }}\right), 2.62 \mathrm{~m}$ (1H, $\left.\mathrm{CH}_{\text {cycloprop. }}\right), 1.58$

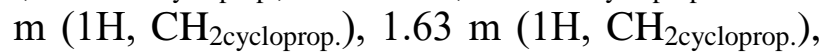
$3.60 \mathrm{~d}\left(2 \mathrm{H}, \mathrm{CH}_{2 \text { eth. }}\right), 4.00 \mathrm{~d}\left(2 \mathrm{H}, \mathrm{CH}_{2 \text { eth. }}\right), 3.00$ $\mathrm{m}\left(1 \mathrm{H}, \mathrm{CH}_{\mathrm{ep}}\right), 2.45 \mathrm{~d}\left(2 \mathrm{H}, \mathrm{CH}_{2 \mathrm{ep}}\right)$. Found, \%: C 65.77, $\mathrm{H}$ 6.52, $\mathrm{Cl}$ 14.37. Calculated, \%: $\mathrm{C}$ 65.41, H 6.29, Cl 14.88.

2-p(nitrophenyl)1-glycidyloxymethylcyclopropane (12). Formula $\mathrm{C}_{13} \mathrm{H}_{15} \mathrm{O}_{4} \mathrm{~N}$. Yield $94 \%$, b.p. $\quad-150-153^{\circ} \mathrm{C} \quad(1 \mathrm{~mm} \quad \mathrm{Hg})$, 
$n_{D}^{20}=1.5250, d_{4}^{20}=1.228, M R_{D}=62.007 .{ }^{1} \mathrm{H}$ NMR spectrum, $\delta$, ppm: $7.85 \mathrm{~m}\left(4 \mathrm{H}_{\text {arom. }}\right), 2.15$ m $\left(1 \mathrm{H}, \mathrm{CH}_{\text {cycloprop. }}\right), 2.68 \mathrm{~m}\left(1 \mathrm{H}, \mathrm{CH}_{\text {cycloprop. }}\right)$, $1.66 \mathrm{~m}$ (1H, $\left.\mathrm{CH}_{2 \text { cycloprop. }}\right), 1.71 \mathrm{~m}(1 \mathrm{H}$, $\left.\mathrm{CH}_{2 \text { cycloprop. }}\right), 3.70 \mathrm{~d}\left(2 \mathrm{H}, \mathrm{CH}_{2 \text { eth. }}\right), 4.10 \mathrm{~d}(2 \mathrm{H}$, $\left.\mathrm{CH}_{2 \text { eth. }}\right), 3.08 \mathrm{~m}\left(1 \mathrm{H}, \mathrm{CH}_{\mathrm{ep}}\right), 2.60 \mathrm{~d}\left(2 \mathrm{H}, \mathrm{CH}_{2 \mathrm{ep}}\right)$. Found, \%: C 62.11, H 6.78, N 5.89. Calculated, $\%$ : C 62.65, H 6.02, N 5.62.

\section{Conclusion}

1. The reaction of carboethoxycarbene with styrene and $p$-substituted styrenes under the conditions of the thermocatalytic decomposition of ethyl diazoacetate has been studied. It has been revealed that the substituents in the investigated styrenes have an essential effect on the activity of the double bond with respect to carboethoxycarbene.

2. The composition and structure of the synthesized compounds have been established. It has been revealed that all synthesized cyclopropane-containing compounds show the various biologically active properties.

\section{References}

1. Row R.D., Shix H.V., Alexandr A.T., Mehl R.A., Presher D.A. Cyclopropenones for Metabolic Targeting and Sequential Bioorthogonal Labeling. J. Am. Chem. Soc. 2017. V. 139. Iss. 21. P. 73707375.

2. Bajaj P., Sreenilayam G., Tyagi V., Fasan R. Gram-Scale Synthesis of Chiral CyclopropaneContaining Drugs and Drug Precursors With Engineered Myoglobin Catalysts Featuring Comple- mentary Stereoselectivity. Angew. Chem. Int. Ed. 2016. V. 55. P. 16110-16114.

3. Epshtein A.E., Dolgii I.E., Leemanov B.E., Skvortcova E.K., Nefyodov O.M. Sintez i bakteritcidnye svoistva chetvertichnykh ammonievykh solei iz 2-alkiltciclopropilmetilbromidov. Izv. AN SSSR. Ser. him. 1978. C. 500-503.

4. Kormachev V.V., Koliamshin O.A., Mitrasov Iu.N., Anisimova E.A. Sintez Fosforsoderzhashchikh Efirov 3-(2,2-Dikhlorvinil)-2,2-Dimetiltciclopropan-karbonovoi Kisloty Zhurn. obshch. himii. 1992. T. 62. Vyp. 3. S. 583.

5. Guliyev K.G., Aliyeva A.M., Ponomaryova G.Z., Nurullayeva D.R., Guliyev A.M. Investigation of Photosensitivity of Copolymer with ( $p$-Vinyl Phenyl)CyclopropylMethylCinnamate. Am. J. Ap. Chem. 2014. V. 3. issue 1. P. 21-24.

6. Guliyev K.G., Mamedli S.B., Nurullayeva D.R., Guliyev A.M. Copolymerization of 2-(4-vinylphenyl)cyclopropylmethyl cinnamate with Glycidyl Methacrylate. Eur. Chem. Bull. 2016. V. 5. Issue 3. P. $108-112$.

7. Guliyev K.G., Mamedli S.B., Gulverdashvili T.D., Guliyev A.M. Photosensitive Copolymers of para(2-Carboxy)cyclopropylStyrene with 2,3-Dimethylbutadiene-1,3. Applied Chem. and Chemical Engineering. 2017. V. 2. Chapter 4. P. 46-53.

8. Guliyev K.G., Rzayeva A.E., Mamedli S.B., Khamedova U.A., Nurullayeva D.R., Guliyev A.M. Synthesis And Polymerization of $p$-(Vinylphenyl)2-Chloromethylcyclopropane. World J. Ress. Rev. 2017. V. 5. Issue 5. P. 1-5.

9. Guliev K.G., Sadygova A.I., Ponomaryova G.Z., Alieva A.M., Gulverdashvili TC.D., Tagiev D.B. Sintez 2-KHlormetil-1-(p-vinilfenil)tciclopropana i ego sopolimerizatciia so stirolom. Zhurn. Pricl. Himii. 2018. T. 91. Vyp. 2. C. 257-262. RJAC. 2018. V. 91. No 2. P. 304-309.

\section{FENIL və $p$-FENIL əVӘZLİ TSIKLOPROPANKARBON TURŞUSUNUN ETIL EFIRLəRINIIN SINTEZI Və ÇEVRILMOLORI}

\section{K.Q.Quliyev, A.E.Rzayeva, A.M.Quliyev}

Etildiazoasetatın termokatalitik parçlanması iştirakında karboetoksikarbenin stirol və p-xlorstirol ilə qarşılıqlı təsir reaksiyası öyrənilmişdir. Aşkar edilmişdir ki, istifadə edilmiş stirollarda olan əvəzedicilərin təbiəti karboetoksikarbenlə reaksiya zamanı ikiqat rabitənin aktivliyinə əhəmiyyətli dərəcədə təsir göstərir. Sintez olunmuş birləşmələrin tərkib və quruluşu təyin edilmişdir. Tədqiqatlar nəticəsində müəyyən edilmişdir ki, stirola para vəziyyətdə birləşmiş əvəzləyicilər karboetoksikarbendə olan ikiqat rabitənin aktivliyinə əhəmiyyətli dərəcədə təsir göstərirlər. Aşkar olunmuşdur ki, stirolun və parastirolların ikqat rabitəsinə karboetoksikarbenin birləşməsi praktiki olaraq əlavə məhsullar alınmadan gedir. Reaksiya, 2-fenil və 2-p-əvəzli-fenil-1-etoksikarboniltsiklopropan (1-4) birləşmələrinin sis- və trans- izormerləri qarışı̆̆ı şəklində və 30:70 nisbətində, tans izomerin alınması üstünlüyü ilə gedir. Sintez edilmiş birləşmələrin tərkib və quruluşları öyrənilmişdir. Məlum olmuşdur ki, bütün sintez edilmiş tsiklopropan tərkibli birləşmələr müxtəlif bioloji aktivliyə malikdirlər. Bundan əlavə, qeyd etmək lazımdır ki, 9-12 birləşmələri ED-20 epoksid qətranı üçün modifikator və qatq1 maddəsi kimi də istifadə oluna bilər.

Açar sözlor: tsiklopropan, p-avazli stirol, karboetoksikarben, qlisidiloksimetiltsiklopropan. 


\title{
СИНТЕЗ ЭТИЛОВЫХ ЭФИРОВ ФЕНИЛ И $\boldsymbol{n}$-ФЕНИЛЗАМЕЩЕННЫХ ЦИКЛОПРОПАНКАРБОНОВЫХ КИСЛОТ И ИХ ПРЕВРАЩЕНИЯ
}

\author{
К.Г.Гулиев, А.Э.Рзаева, А.М.Гулиев
}

Изучена реакция карбоэтоксикарбена со стиролом и $n$-замещенных стиролов в условиях термокаталитического разложения этилдиазоацетата. Показано, что заместители в исследуемых стиролах оказывают существенное влияние на активность двойной связи по отношению к карбоэтоксикарбену. В результате исследования установлено, что заместители в пара положении стирола оказывают существенное влияние на активность двойной связи по отношению карбоэтоксикарбену. Выявлено, что карбоэтоксикарбен присоединяется к двойной связи стирола и п-стиролов практически без образования побочных продуктов. В результате реакции был получен 2-фенил и 2-n-замещенныхфенил-1-этоксикарбонил циклопропан $(1-4)$ в виде смеси ицс- и трансизомеров в соотношении $\sim 30: 70$ с преимущественным образованием транс изомера. Установлены состав и структуры синтезированных соединений. Выявлено, что все синтезированные циклопропансодержащие соединения проявляют различные биологически активные свойства. Кроме этого соединения 9-12 можно использовать в качестве модификатора и разбавителя для эпоксидной смолы ЭД-20.

Ключевые слова: ијиклопропан, паразамещенный стирол, карбоэтоксикарбена, глищидилоксиметилииклопропан. 